\title{
NLO fragmentation functions of heavy quarks into heavy quarkonia
}

\author{
Xu-Chang Zheng, ${ }^{1, *}$ Chao-Hsi Chang, ${ }^{2,3,4, \dagger}$ and Xing-Gang $\mathrm{Wu}^{1, *}$ \\ ${ }^{1}$ Department of Physics, Chongqing University, Chongqing 401331, People's Republic of China \\ ${ }^{2}$ Key Laboratory of Theoretical Physics, Institute of Theoretical Physics, Chinese Academy of Sciences, \\ Beijing 100190, China \\ ${ }^{3}$ School of Physical Sciences, University of Chinese Academy of Sciences, Beijing 100049, China \\ ${ }^{4}$ CCAST (World Laboratory), Beijing 100190, China
}

(Received 18 May 2019; published 9 July 2019)

\begin{abstract}
In this paper we derive the next-to-leading-order (NLO) fragmentation function for a heavy quark, either charm or bottom, into a heavy quarkonium $J / \Psi$ or $\Upsilon$. The ultraviolet divergences in the real corrections are removed through operator renormalization, which is performed in the modified minimal subtraction scheme. We then obtain the NLO fragmentation function at an initial factorization scale, e.g., $\mu_{F}=3 m_{c}$ for $c \rightarrow J / \Psi$ and $\mu_{F}=3 m_{b}$ for $b \rightarrow \Upsilon$, which can be evolved to any scale via the use of the DokshitzerGribov-Lipatov-Altarelli-Parisi equation. As an initial application of these fragmentation functions, we study $J / \Psi(\Upsilon)$ production at a high-luminosity $e^{+} e^{-}$collider running at an energy around the $Z$ pole, which could be a suitable platform for testing the fragmentation function.
\end{abstract}

DOI: 10.1103/PhysRevD.100.014005

\section{INTRODUCTION}

Since the observation of the $J / \Psi$ meson, heavy quarkonia have attracted great interest from theorists and experimentalists. Due to the fact that the constituent quark $(Q)$ and antiquark $(\bar{Q})$ of a heavy quarkonium are heavy, i.e, $m_{Q} \gg \Lambda_{\mathrm{QCD}}$, its production rate and decay width involve both perturbative and nonperturbative aspects of QCD. It then provides a good platform for testing various QCD factorization theories. Within the framework of the nonrelativistic QCD (NRQCD) factorization theory [1], the production cross section of a heavy quarkonium via the collision of two incident particles $A$ and $B$ can be written as

$$
\begin{aligned}
& d \sigma(A+B \rightarrow H+X) \\
& =\sum_{n} d \tilde{\sigma}(A+B \rightarrow(Q \bar{Q})[n]+X)\left\langle Q_{n}^{H}\right\rangle,
\end{aligned}
$$

where $H$ denotes the produced heavy quarkonium, and $d \tilde{\sigma}$ is the production cross section for the perturbative state $(Q \bar{Q})[n]$ with quantum numbers $n$, which is calculable and can be expanded in powers of the strong coupling

\footnotetext{
zhengxc@cqu.edu.cn

thangzx@itp.ac.cn

*wuxg@cqu.edu.cn
}

Published by the American Physical Society under the terms of the Creative Commons Attribution 4.0 International license. Further distribution of this work must maintain attribution to the author(s) and the published article's title, journal citation, and DOI. Funded by SCOAP. constant $\alpha_{s} \cdot\left\langle Q_{n}^{H}\right\rangle$ denotes the nonperturbative but universal NRQCD matrix element, which is proportional to the transition probability of the perturbative state $(Q \bar{Q})[n]$ into the hadron state $H$.

The NRQCD factorization formulism has been used to deal with quarkonium production at $e^{+} e^{-}$and hadron colliders, and most of the calculations have been performed up to next-to-leading-order (NLO) accuracy [2,3]. In some cases, there are large logarithms in the short-distance part $d \tilde{\sigma}$. For instance, there are large logarithms in powers of $\ln \left(\sqrt{s} / m_{Q}\right)$ for heavy quarkonium production at $e^{+} e^{-}$colliders, with $\sqrt{s}$ being the center-of-mass collision energy, or there are large logarithms in powers of $\ln \left(p_{T} / m_{Q}\right)$ in the high- $p_{T}$ region (with $p_{T}$ being the transverse momentum of the quarkonium) at hadron colliders. Those large logarithms may spoil the convergence of the perturbative expansion, leading to the unreliable perturbative QCD (pQCD) predictions. As a solution, it has been pointed out that these logarithms mainly come from the emission of collinear gluons, which can be systematically resummed through the Dokshitzer-GribovLipatov-Altarelli-Parisi (DGLAP) evolution equation [4].

We shall take heavy quarkonium production at an $e^{+} e^{-}$ collider as an explicit example to explain this idea; in particular, we shall give the results for the heavy quark to heavy quarkonium fragmentation function up to NLO accuracy. The fragmentation function gives the probability that a parton will split into the desired hadron plus other partons. The fragmentation function of a hadron composed of only heavy quarks can be calculated using pQCD theory. The $Z$ boson decays $Z \rightarrow J / \Psi+X$ and $Z \rightarrow \Upsilon+X$ have 
been studied up to the NLO level using the complete pQCD approach [5], and in the present paper we will study these processes but using the fragmentation approach. Compared with the complete $\mathrm{pQCD}$ approach, even though the fragmentation approach neglects some powersuppressed terms, it resums the large collinear logarithms more conveniently via the use of the DGLAP evolution equation and achieves more reliable predictions in specific kinematic regions.

In the pQCD factorization theory, the production cross section of heavy quarkonium at an $e^{+} e^{-}$collider can be factorized as

$$
\begin{aligned}
d \sigma\left(e^{+} e^{-} \rightarrow H(p)+X\right)= & \sum_{i} d \hat{\sigma}\left(e^{+} e^{-} \rightarrow i(p / z)+X, \mu_{F}\right) \\
& \otimes D_{i \rightarrow H}\left(z, \mu_{F}\right)+\mathcal{O}\left(m_{Q}^{2} / s\right),
\end{aligned}
$$

where the sum extends over all of the parton types, $d \hat{\sigma}$ denotes the partonic cross section (coefficient function), and $D_{i \rightarrow H}\left(z, \mu_{F}\right)$ denotes the fragmentation function (decay function) for the parton $i$ into heavy quarkonium $H$ with longitudinal momentum fraction $z . \mu_{F}$ is the factorization scale which separates the energy scales of the two parts. In order to prevent large logarithms from appearing in $d \hat{\sigma}, \mu_{F}$ is usually set as $\mu_{F}=\mathcal{O}(\sqrt{s})$.

The pQCD factorization formula (2) was first suggested by Collins and Soper for the inclusive production of a light hadron [6], and the proof of the pQCD factorization formula for the case of quarkonium production was given by Nayak, Qiu, and Sterman [7]. One recent achievement in using $\mathrm{pQCD}$ factorization for quarkonium production is the derivation of the next-to-leading-power contribution, which comes from double-parton fragmentation [8-11]. The fragmentation function $D_{i \rightarrow H}\left(z, \mu_{F}\right)$ contains nonperturbative information, which is calculable through NRQCD factorization (or the Mandelstam formulation [12] under the instantaneous approximation) $[13,14]$, e.g., $D_{i \rightarrow H}\left(z, \mu_{F}\right)$ can be factorized as

$$
D_{i \rightarrow H}\left(z, \mu_{F}\right)=\sum_{n} d_{i \rightarrow(Q \bar{Q})[n]}\left(z, \mu_{F}\right)\left\langle Q_{n}^{H}\right\rangle,
$$

where $d_{i \rightarrow(Q \bar{Q})[n]}\left(z, \mu_{F}\right)$ is the short-distance coefficient, which contains logarithms of $\mu_{F} / m_{Q}$. To avoid such kind of large logarithms, one can first calculate the fragmentation function at some initial factorization scale that is of order $\mathcal{O}\left(m_{Q}\right)$, and then evolve it to a higher factorization scale by using the DGLAP evolution equation [15-17],

$$
\begin{aligned}
& \frac{d}{d \ln \mu_{F}^{2}} D_{i \rightarrow H}\left(z, \mu_{F}\right) \\
& =\frac{\alpha_{s}\left(\mu_{F}\right)}{2 \pi} \sum_{j} \int_{z}^{1} \frac{d y}{y} P_{j i}\left(y, \alpha_{s}\left(\mu_{F}\right)\right) D_{j \rightarrow H}\left(z / y, \mu_{F}\right),
\end{aligned}
$$

where $P_{j i}$ are splitting functions, which can be expanded in perturbative series as,

$P_{j i}\left(y, \alpha_{s}\left(\mu_{F}\right)\right)=\frac{\alpha_{s}\left(\mu_{F}\right)}{2 \pi} P_{j i}^{(0)}(y)+\frac{\alpha_{s}^{2}\left(\mu_{F}\right)}{(2 \pi)^{2}} P_{j i}^{(1)}(y)+\mathcal{O}\left(\alpha_{s}^{3}\right)$.

For the quark-to-quark case, the LO coefficient is

$$
P_{Q Q}^{(0)}(y)=C_{F}\left[\frac{1+y^{2}}{(1-y)_{+}}+\frac{3}{2} \delta(1-y)\right],
$$

where $C_{F}=4 / 3$ for the $S U(3)_{c}$ group. The NLO coefficient $P_{Q Q}^{(1)}(y)$ is too lengthy to be presented here, and can be found in Refs. [18-20].

The LO fragmentation function for a heavy quark to $J / \Psi$ or $\Upsilon$ was first calculated by Braaten and Cheung in 1993 [21], where the LO fragmentation function was derived from an LO calculation of the process $Z \rightarrow J / \Psi+c+\bar{c}$. Subsequently, Ma calculated the LO fragmentation functions [22] by using the gauge-invariant definition suggested by Collins and Soper. In Ref. [23], the authors calculated the NLO corrections to the transverse-momentumdependent fragmentation functions for a heavy quark to $J / \Psi$ and $\Upsilon$, which is however not convenient for practical applications. Recently, the NLO fragmentation function for a gluon into heavy quarkonium was determined in Refs. [24-27]. In the present paper, we shall give the fragmentation functions for a heavy quark into $J / \Psi$ and $\Upsilon$ up to the NLO level.

The remaining parts of the paper are organized as follows. In Sec. II, we present the LO fragmentation function for a heavy quark into the heavy quarkonium $J / \Psi$ or $\Upsilon$. In Sec. III, we present the NLO fragmentation functions $D_{c \rightarrow J / \Psi}$ and $D_{b \rightarrow \mathrm{r}}$, in which the renormalization is carried out by using the conventional $\overline{\mathrm{MS}}$ scheme. In Sec. IV, we apply these NLO fragmentation functions to $J / \Psi$ and $\Upsilon$ production at a super $Z$ factory. Section V is reserved for a summary.

\section{THE LO FRAGMENTATION FUNCTION}

Before carrying out the calculation for the fragmentation function, we first give a brief introduction of the gaugeinvariant fragmentation function suggested by Collins and Soper [6]. We adopt dimensional regularization to regularize the infrared (IR) and ultraviolet (UV) divergences, and work in $d=4-2 \epsilon$-dimensional space-time.

The light-cone coordinates are conventionally adopted to define the fragmentation function, where a vector $V^{\mu}$ in $d$ dimensions is expressed as $V^{\mu}=\left(V^{+}, V^{-}, \mathbf{V}_{\perp}\right)=$ $\left(\left(V^{0}+V^{d-1}\right) / \sqrt{2},\left(V^{0}-V^{d-1}\right) / \sqrt{2}, \mathbf{V}_{\perp}\right)$. The scalar product of two vectors $V$ and $W$ then becomes $V \cdot W=$ $V^{+} W^{-}+V^{-} W^{+}-\mathbf{V}_{\perp} \cdot \mathbf{W}_{\perp}$. The gauge-invariant fragmentation function for the heavy quark $Q$ into a spin-triplet and color-singlet quarkonium $H$ in $d=4-2 \epsilon$ dimensions is defined as 


$$
\begin{aligned}
D_{Q \rightarrow H}(z)= & \frac{z^{d-3}}{2 \pi} \sum_{X} \int d x^{-} e^{-i P^{+} x^{-} / z} \\
& \times \frac{1}{N_{c}} \operatorname{Tr}_{\text {color }} \frac{1}{4} \operatorname{Tr}_{\text {Dirac }}\left\{\gamma^{+}\left\langle 0\left|\Psi(0) \overline{\mathcal{P}} \exp \left[i g_{s} \int_{0}^{\infty} d y^{-} A_{a}^{+}\left(0^{+}, y^{-}, 0_{\perp}\right) t t_{a}^{\perp}\right]\right| H\left(P^{+}, 0_{\perp}\right)+X\right\rangle\right. \\
& \left.\times\left\langle H\left(P^{+}, 0_{\perp}\right)+X\left|\mathcal{P} \exp \left[-i g_{s} \int_{x^{-}}^{\infty} d y^{-} A_{a}^{+}\left(0^{+}, y^{-}, 0_{\perp}\right) t_{a}^{\perp}\right] \bar{\Psi}(x)\right| 0\right\rangle\right\}
\end{aligned}
$$

where $\Psi$ and $A_{a}^{\mu}$ are the quark field and gluon field, respectively $t^{a}$ is the color matrix, $\mathcal{P}$ implies the path ordering, and $z \equiv P^{+} / K^{+}$is the longitudinal momentum fraction carried from the incident heavy quark $Q$. This definition is carried out in a reference frame where the quarkonium $H$ carries no transverse momentum, i.e., $P^{\mu}=\left(P^{+}, P^{-}=\right.$ $\left.m_{H}^{2} / 2 P^{+}, 0_{\perp}\right)$. It is convenient to introduce a light-like vector $n^{\mu}$, which has the value $n^{\mu}=\left(0,1,0_{\perp}\right)$ in the reference frame where the fragmentation function is defined. In this frame, the plus component of the momentum $p$ can be expressed as $p^{+}=p \cdot n$, and $z=P \cdot n / K \cdot n$. The Feynman rules for the fragmentation function can be directly derived from the above definition [28].

The fragmentation function (7) is gauge independent. For our practical treatment, we work in the usual Feynman gauge. To derive the fragmentation function $D_{Q \rightarrow H}$, we first calculate the fragmentation function for the production of a free on-shell $Q \bar{Q}$ state with the quantum numbers ${ }^{3} S_{1}^{[1]}$ (i.e, $\left.D_{Q \rightarrow Q \bar{Q}\left[{ }^{3} S_{1}^{[1]}\right]}\right)$, where the superscript "[1]" denotes the $Q \bar{Q}$ state is a color singlet. Then, $D_{Q \rightarrow H}$ is obtained by replacing the NRQCD matrix element of a free $Q \bar{Q}$ state $\left.\left[\left\langle\mathcal{O} Q \bar{Q}{ }^{[3} S_{1}^{[1]}\right]\left({ }^{3} S_{1}^{[1]}\right)\right\rangle\right]$ with the matrix element of the quarko$\operatorname{nium}\left(\left\langle\mathcal{O}^{H}\left({ }^{3} S_{1}^{[1]}\right)\right\rangle\right)$.
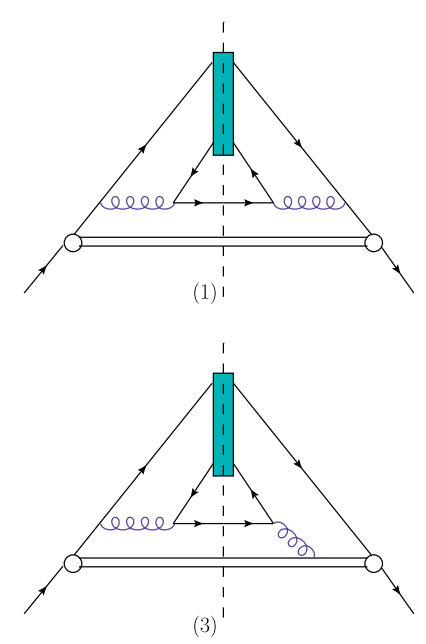

FIG. 1. The LO cut diagrams for the fragmentation function $D_{Q \rightarrow Q \bar{Q}\left[{ }^{3} S_{1}^{[1]}\right]}$. The double line stands for the Wilson line which ensures the gauge invariance of the squared amplitude.
At the LO level, we need to deal with the process $Q(K) \rightarrow Q \bar{Q}\left[{ }^{3}{ }^{[1]}\right]\left(p_{1}\right)+Q\left(p_{2}\right)$. As shown in Fig. 1, there are four LO cut diagrams which contribute to $D_{Q \rightarrow Q \bar{Q}}\left[{ }^{3} S_{1}^{[1]}\right]$. The four squared amplitudes from the four cut diagrams are

$$
\begin{aligned}
\mathcal{A}_{1}= & \operatorname{tr}\left[\left(\not \not_{2}+m_{Q}\right)\left(i g_{s} \gamma^{\mu} t^{a}\right) \Pi \Lambda_{1}\left(i g_{s} \gamma_{\mu} t^{a}\right)\right. \\
& \cdot \frac{i}{\not p_{1}+\not p_{2}-m_{Q}+i \epsilon} \not h \frac{-i}{\not \not_{1}+\not p_{2}-m_{Q}-i \epsilon} \\
& \left.\cdot\left(-i g_{s} \gamma^{\nu} t^{b}\right) \bar{\Pi} \Lambda_{1}\left(-i g_{s} \gamma_{\nu} t^{b}\right)\right] \\
& \cdot \frac{-i}{\left(p_{1} / 2+p_{2}\right)^{2}+i \epsilon} \frac{i}{\left(p_{1} / 2+p_{2}\right)^{2}-i \epsilon},
\end{aligned}
$$

$\mathcal{A}_{2}=\operatorname{tr}\left[\left(\not 2_{2}+m_{Q}\right)\left(i g_{s} \gamma^{\mu} t^{a}\right) \Pi \Lambda_{1}\right.$

$$
\begin{aligned}
& \cdot \frac{i}{\left(-p_{1} / 2-p_{2}\right) \cdot n+i \epsilon}\left(i g_{s} n_{\mu} t^{a}\right) \not h \\
& \left.\cdot \frac{-i}{\not 1+\not 22-m_{Q}-i \epsilon}\left(-i g_{s} \gamma^{\nu} t^{b}\right) \bar{\Pi} \Lambda_{1}\left(-i g_{s} \gamma_{\nu} t^{b}\right)\right] \\
& \cdot \frac{-i}{\left(p_{1} / 2+p_{2}\right)^{2}+i \epsilon} \frac{i}{\left(p_{1} / 2+p_{2}\right)^{2}-i \epsilon},
\end{aligned}
$$$$
\mathcal{A}_{3}=\operatorname{tr}\left[\left(\not \not_{2}+m_{Q}\right)\left(i g_{s} \gamma^{\mu} t^{a}\right) \Pi \Lambda_{1}\left(i g_{s} \gamma_{\mu} t^{a}\right)\right.
$$$$
\frac{i}{\not p 1+\not p 2-m_{Q}+i \epsilon} \not h\left(-i g_{s} n_{\nu} t^{b}\right)
$$$$
\left.\cdot \frac{-i}{\left(-p_{1} / 2-p_{2}\right) \cdot n-i \epsilon} \bar{\Pi} \Lambda_{1}\left(-i g_{s} \gamma_{\nu} t^{b}\right)\right]
$$$$
\cdot \frac{-i}{\left(p_{1} / 2+p_{2}\right)^{2}+i \epsilon} \frac{i}{\left(p_{1} / 2+p_{2}\right)^{2}-i \epsilon} \text {, }
$$

$$
\begin{aligned}
\mathcal{A}_{4}= & \operatorname{tr}\left[\left(\not \not p_{2}+m_{Q}\right)\left(i g_{s} \gamma^{\mu} t^{a}\right) \Pi \Lambda_{1}\right. \\
& \cdot \frac{i}{\left(-p_{1} / 2-p_{2}\right) \cdot n+i \epsilon}\left(i g_{s} n_{\mu} t^{a}\right) \not h\left(-i g_{s} n_{\nu} t^{b}\right) \\
& \left.\cdot \frac{-i}{\left(-p_{1} / 2-p_{2}\right) \cdot n-i \epsilon} \bar{\Pi} \Lambda_{1}\left(-i g_{s} \gamma_{\nu} t^{b}\right)\right] \\
& \cdot \frac{-i}{\left(p_{1} / 2+p_{2}\right)^{2}+i \epsilon} \frac{i}{\left(p_{1} / 2+p_{2}\right)^{2}-i \epsilon}
\end{aligned}
$$

Here $\Pi$ denotes the spin projector, and for the ${ }^{3} S_{1}$ state 


$$
\Pi=-\frac{1}{2 \sqrt{2 m_{Q}}} \not\left(p_{1}\right)\left(\not p_{1}+2 m_{Q}\right),
$$

and $\bar{\Pi} \equiv \gamma^{0} \Pi^{\dagger} \gamma^{0} . \Lambda_{1}$ is color-singlet projector,

$$
\Lambda_{1}=\frac{1}{\sqrt{3}}
$$

where 1 is the $S U_{c}(3)$ unit matrix.

Then we obtain the total squared amplitude at the LO level,

$$
\mathcal{A}_{\text {Born }}=\sum_{j=1}^{4} \mathcal{A}_{j}=\frac{2 C_{F}^{2} g_{s}^{4} K \cdot n}{(2-z)^{2} m_{Q}} \sum_{j=2}^{4} \frac{a_{j} m_{Q}^{2(j-2)}}{\left(s_{1}-m_{Q}^{2}\right)^{j}},
$$

where $s_{1}=\left(p_{1}+p_{2}\right)^{2}$ is the invariant mass of the final $Q \bar{Q}\left[{ }^{3} S_{1}^{[1]}\right]+Q$, and the coefficients $a_{j=2,3,4}$ are

$$
\begin{aligned}
a_{2}= & (1-z)\left[\left(d^{3}-17 d^{2}+100 d-156\right) z^{2}-4\left(d^{3}-13 d^{2}\right.\right. \\
& \left.+56 d-84) z+4\left(d^{3}-9 d^{2}+28 d-28\right)\right], \\
a_{3}= & 8(z-2)\left[\left(d^{2}-9 d+16\right) z^{2}-2\left(d^{2}-5 d+16\right) z+16\right], \\
a_{4}= & -64(d-1)(z-2)^{2} .
\end{aligned}
$$

The differential phase space for the LO fragmentation function is

$$
d \phi_{\mathrm{Born}}=\frac{d p_{2}^{+}}{2 p_{2}^{+}} \frac{d^{d-2} \mathbf{p}_{2 \perp}}{(2 \pi)^{d-2}} 2 \pi \delta\left(K^{+}-p_{1}^{+}-p_{2}^{+}\right),
$$

where the $\delta$ function comes from the final cut of the eikonal line. The integration over $p_{2}^{+}$can be carried out with the $\delta$ function. The integration over the angles of $\mathbf{p}_{2 \perp}$ is trivial and can be carried out easily. Then we have

$$
\begin{aligned}
d \phi_{\mathrm{Born}}= & \frac{z^{-1+\epsilon}(1-z)^{-\epsilon}}{2(4 \pi)^{1-\epsilon} \Gamma(1-\epsilon) K \cdot n} \\
& \times\left(s_{1}-\frac{4 m_{Q}^{2}}{z}-\frac{m_{Q}^{2}}{1-z}\right)^{-\epsilon} d s_{1} .
\end{aligned}
$$

The range of $s_{1}$ is from $\left[4 m_{Q}^{2} / z+m_{Q}^{2} /(1-z)\right]$ to $+\infty$.

The LO fragmentation function for $Q \rightarrow Q \bar{Q}\left[{ }^{3} S_{1}^{[1]}\right]$ can be obtained as

$$
D_{Q \rightarrow Q \bar{Q}\left[\left.{ }^{[S}\right|_{1} ^{[1]}\right]}^{\mathrm{LO}}(z)=N_{\mathrm{CS}} \int d \phi_{\mathrm{Born}} \mathcal{A}_{\mathrm{Born}},
$$

where $N_{\mathrm{CS}}=z^{1-2 \epsilon} / 8 \pi N_{c}$ is an overall factor. Performing the integration over $s_{1}$, we obtain

$$
\begin{aligned}
D_{Q \rightarrow Q \bar{Q}\left[{ }^{3} S_{1}^{[1]}\right]}^{\mathrm{LO}}(z)= & \frac{C_{F}^{2} \alpha_{s}^{2} z(1-z)(4 \pi)^{\epsilon} \Gamma(1+\epsilon)}{2 N_{c}(2-z)^{4+2 \epsilon} m_{Q}^{3+2 \epsilon}} \\
& \times\left[a_{2}+a_{3} \frac{(1+\epsilon) z(1-z)}{2(2-z)^{2}}\right. \\
& \left.+a_{4} \frac{(2+\epsilon)(1+\epsilon) z^{2}(1-z)^{2}}{6(2-z)^{4}}\right] .
\end{aligned}
$$

Setting $d=4$, we obtain

$$
\begin{aligned}
D_{Q \rightarrow Q \bar{Q}\left[{ }^{[3} S_{1}^{[1]}\right]}^{\mathrm{LO}}(z)= & \frac{32 \alpha_{s}^{2} z(1-z)^{2}}{27(2-z)^{6} m_{Q}^{3}} \\
& \times\left(5 z^{4}-32 z^{3}+72 z^{2}-32 z+16\right) \\
& \times \frac{\left.\left\langle\mathcal{O} Q \bar{Q}{ }^{[3} S_{1}^{[1]}\right]\left({ }^{3} S_{1}^{[1]}\right)\right\rangle}{6 N_{c}} .
\end{aligned}
$$

Here, the LO fragmentation function for the free $Q \bar{Q}\left[{ }^{3} S_{1}^{[1]}\right]$ state has been written in the factorization form by using the fact that

$$
\left.\left\langle\mathcal{O}^{Q} \bar{Q}^{[3} S_{1}^{[1]}\right]\left({ }^{3} S_{1}^{[1]}\right)\right\rangle=2(d-1) N_{c}
$$

at the order $\alpha_{s}^{0}$ with the normalization of the NRQCD matrix element from Ref. [1]. Then the LO fragmentation function for the quarkonium can be obtained by replacing $\left\langle\mathcal{O}^{Q} \bar{Q}^{\left[{ }^{[3}{ }_{1}^{[1]}\right]}\left({ }^{3} S_{1}^{[1]}\right)\right\rangle$ with $\left\langle\mathcal{O}^{H}\left({ }^{3} S_{1}^{[1]}\right)\right\rangle$. Under the leading nonrelativistic approximation, the matrix element $\left\langle\mathcal{O}^{H}\left({ }^{3} S_{1}^{[1]}\right)\right\rangle$ can be expressed by the radial wave function at the origin for the quarkonium $H$, i.e.,

$$
\left\langle\mathcal{O}^{H}\left({ }^{3} S_{1}^{[1]}\right)\right\rangle \approx(d-1) N_{c}\left|R_{S}(0)\right|^{2} /(2 \pi) .
$$

Finally, the LO fragmentation function for the ${ }^{3} S_{1}$ quarkonium state takes the form

$$
\begin{aligned}
D_{Q \rightarrow H}^{\mathrm{LO}}(z)= & \frac{8 \alpha_{s}^{2} z(1-z)^{2}\left|R_{S}(0)\right|^{2}}{27 \pi(2-z)^{6} m_{Q}^{3}} \\
& \times\left(5 z^{4}-32 z^{3}+72 z^{2}-32 z+16\right),
\end{aligned}
$$

which is exactly the same as that of Ref. [21].

\section{THE NLO CORRECTION TO THE FRAGMENTATION FUNCTION}

At the NLO level, we need to deal with the virtual and real corrections to the LO terms. It is hard to give the analytic expressions for those NLO terms. In the following subsections, we shall give some explanations of how to deal with the virtual and real corrections.

In our calculations we use the FEYNCALC package $[29,30]$ to carry out the color and Dirac traces, and the \$APART package [31] and the FIRE package [32] are used to 

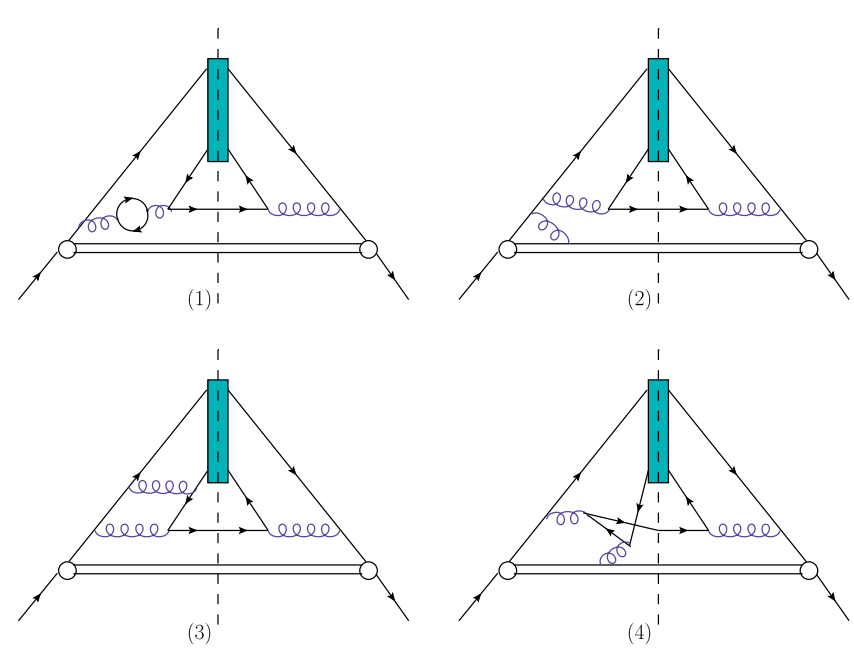

FIG. 2. Four typical virtual corrections to the fragmentation function $D_{Q \rightarrow Q \bar{Q}\left[{ }^{[} S_{1}^{[1]}\right]}$. The double line stands for the Wilson line which ensures the gauge invariance of the squared amplitude.

do partial fraction and integration-by-parts reduction. The master integrals are calculated using the LOOPToOLS package [33]. As a subtle point, there are some master integrals that contain an eikonal propagator and cannot be calculated using LoopTools, and we adopt the method introduced in Ref. [24] to deal with these master integrals.

\section{A. The virtual corrections}

At the NLO level, the virtual corrections come from the cut diagrams containing a loop on either side of the cut. Four typical virtual corrections are shown in Fig. 2. The differential phase space of the virtual corrections is the same as that of the LO one, e.g., Eq. (15). The virtual corrections can be obtained as

$$
D_{Q \rightarrow Q \bar{Q}\left[S_{1}^{\left[S_{1}^{[1]}\right]}\right.}^{\text {virtual }}(z)=N_{\mathrm{CS}} \int d \phi_{\text {Born }} \mathcal{A}_{\text {virtual }},
$$

where $\mathcal{A}_{\text {virtual }}$ is the squared amplitude for the virtual corrections.

We adopt the method of regions [34] to calculate the fragmentation function. With this method we only need to calculate the contributions from the hard region, as the Coulomb divergences which come from the potential region do not appear in the calculations. The hard-region contributions are then obtained by expanding the relative velocity between the produced $Q$ and $\bar{Q}$ before the loop integration. Thus, in the calculation we only need to set the relative momentum to zero before the loop integration [34].

\section{B. The real corrections}

At the NLO level, the real corrections come from the fragmentation process emitting an extra gluon, i.e., we need to deal with the process $Q(K) \rightarrow Q \bar{Q}\left[{ }^{3} S_{1}^{[1]}\right]\left(p_{1}\right)+Q\left(p_{2}\right)+$ $g\left(p_{3}\right)$. The cut diagrams for the real corrections can be
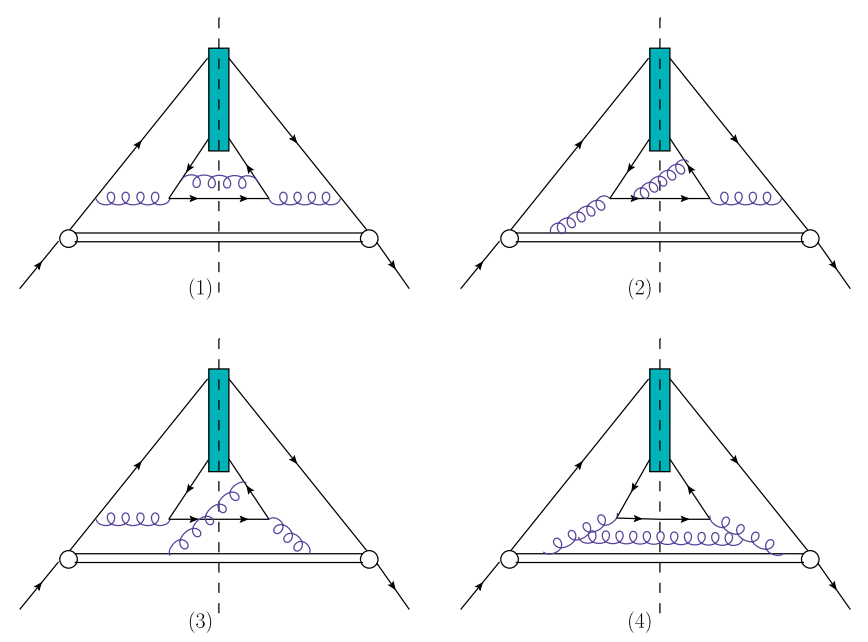

FIG. 3. Four typical real corrections to the fragmentation function $D_{Q \rightarrow Q \bar{Q}\left[{ }^{3} S_{1}^{[1]}\right]}$. The double line stands for the Wilson line which ensures the gauge invariance of the squared amplitude.

obtained from the LO cut diagrams by adding a gluon line crossing the cut. Four typical real correction cut diagrams are shown in Fig. 3.

The differential phase space for the real correction is

$$
d \phi_{\text {real }}=2 \pi \delta\left(K^{+}-\sum_{i=1}^{3} p_{i}^{+}\right) \prod_{i=2,3} \frac{d p_{i}^{+}}{2 p_{i}^{+}} \frac{d^{d-2} \mathbf{p}_{i \perp}}{(2 \pi)^{d-2}} .
$$

The real corrections can be obtained as

$$
D_{Q \rightarrow Q \bar{Q}\left[S_{1}^{\left[S_{1}^{[1]}\right]}\right.}^{\text {real }}(z)=N_{\mathrm{CS}} \int d \phi_{\text {real }} \mathcal{A}_{\text {real }}
$$

where $\mathcal{A}_{\text {real }}$ denotes the squared amplitude for the real corrections. As for the real corrections, the IR divergences come from the limits $p_{3} \rightarrow 0$ and $p_{3} \cdot n \rightarrow 0$, and the UV divergences come from the limit $\left|\mathbf{p}_{3 \perp}\right| \rightarrow \infty$. We adopt the method of Ref. [24] to extract these divergences. Following this method, the real corrections can be represented as

$$
\begin{aligned}
D_{Q \rightarrow Q \bar{Q}[n]}^{\text {real }}(z)= & N_{\mathrm{CS}} \int d \phi_{\text {real }}\left(\mathcal{A}_{\text {real }}-\mathcal{A}_{S}\right) \\
& +N_{\mathrm{CS}} \int d \phi_{\text {real }} \mathcal{A}_{S},
\end{aligned}
$$

where $\mathcal{A}_{S}$ denotes the subtraction term, which has the same singularities as the squared amplitude of the real corrections. The first term on the right-hand side of Eq. (26) is finite and can be directly calculated in four dimensions. The integral of the subtraction term is divergent and should be calculated in $d$ dimensions.

The subtraction term can be constructed according to the singularity behavior of the squared amplitude of the real corrections. More explicitly, the squared amplitude for the real corrections can be written as 


$$
\begin{aligned}
\mathcal{A}_{\text {real }}= & \frac{b_{1}\left(s_{1}, z\right)}{(1-y)\left(s-m_{Q}^{2}\right)}+\frac{b_{2}\left(s_{1}, z\right)}{(1-y)\left(s_{2}-m_{Q}^{2}\right)}+\frac{b_{3}\left(s_{1}, z\right)}{(1-y) s_{3}}+\frac{c_{1}\left(s_{1}, z, y\right)}{s-m_{Q}^{2}}+\frac{c_{2}\left(s_{1}, z, y\right) p_{1} \cdot p_{3}}{\left(s-m_{Q}^{2}\right)^{2}}+\frac{c_{3}\left(s_{1}, z, y\right)}{s_{2}-m_{Q}^{2}} \\
& +\frac{c_{4}\left(s_{1}, z, y\right) p_{2} \cdot p_{3}}{\left(s_{2}-m_{Q}^{2}\right)^{2}}+\frac{c_{5}\left(s_{1}, z, y\right)}{s_{3}}+\frac{c_{6}\left(s_{1}, z, y\right) p_{1} \cdot p_{3}}{s_{3}^{2}}+\frac{d_{1}\left(s_{1}, z\right)(1-u)\left(s_{1}-m_{Q}^{2}\right)}{u t_{1}\left(s-m_{Q}^{2}\right)} \\
& +\frac{d_{2}\left(s_{1}, z\right)(1-u)\left(s_{1}-m_{Q}^{2}\right)}{2 u t_{1} s_{3}}+\frac{d_{3}\left(s_{1}, z\right)(1-u)\left(s_{1}-m_{Q}^{2}\right)^{2}}{2 u t_{1} s_{3}\left(s-m_{Q}^{2}\right)}+\frac{d_{4}\left(s_{1}, z\right)\left(s_{1}-m_{Q}^{2}\right)^{2}}{2 u t_{2}\left(s-m_{Q}^{2}\right) s_{3}} \\
& +\frac{d_{5}\left(s_{1}, z\right)\left(s_{1}-m_{Q}^{2}\right)}{u t_{2}\left(s-m_{Q}^{2}\right)}+\frac{d_{6}\left(s_{1}, z\right)\left(s_{1}-m_{Q}^{2}\right)}{2 u t_{2} s_{3}}+\frac{g\left(s_{1}, z\right)\left(s_{1}-m_{Q}^{2}\right)^{2}}{2 u\left(s-m_{Q}^{2}\right) s_{3}}+\frac{h\left(s_{1}, z\right)}{t_{2}^{2}}+\mathcal{A}_{\text {real }}^{\text {finite }},
\end{aligned}
$$

where the Lorentz invariants are defined as follows:

$$
\begin{array}{rlrl}
y & =\frac{\left(p_{1}+p_{2}\right) \cdot n}{\left(p_{1}+p_{2}+p_{3}\right) \cdot n}, & u=\frac{p_{3} \cdot n}{\left(p_{2}+p_{3}\right) \cdot n}, \\
s & =\left(p_{1}+p_{2}+p_{3}\right)^{2}, & s_{2}=\left(p_{1} / 2+p_{3}\right)^{2}, \\
s_{3} & =\left(p_{1} / 2+p_{2}+p_{3}\right)^{2}, & t_{1}=2 p_{1} \cdot p_{3}, \\
t_{2} & =2 p_{2} \cdot p_{3} . & &
\end{array}
$$

$\mathcal{A}_{\text {real }}^{\text {finite }}$ stands for the terms that are finite after the phase-space integration. There is neither a $1 / t_{1}^{2}$ term nor a $1 / t_{1} t_{2}$ term in $\mathcal{A}_{\text {real }}$ because those terms are canceled after summing all of the terms of the real corrections. The subtraction term $\mathcal{A}_{\mathrm{S}}$ can then be constructed as follows:

$$
\begin{aligned}
\mathcal{A}_{\mathrm{S}}= & \frac{b_{1}\left(s_{1}, z\right)}{(1-y)\left(s-m_{Q}^{2}\right)}+\frac{b_{2}\left(s_{1}, z\right)}{(1-y)\left(s_{2}-m_{Q}^{2}\right)}+\frac{b_{3}\left(s_{1}, z\right)}{(1-y) s_{3}}+\frac{c_{1}\left(s_{1}, z, y\right)}{s} \\
& +\frac{c_{2}\left(s_{1}, z, y\right)}{s^{2}}\left[p_{1} \cdot p_{3}-\frac{z}{2 y}\left(1-\frac{2}{y}\right) s_{1}-\frac{1-y}{2 y}\left(s_{1}+3 m_{Q}^{2}\right)\right]+\frac{c_{3}\left(s_{1}, z, y\right)}{s_{2}} \\
& +\frac{c_{4}\left(s_{1}, z, y\right)}{s_{2}^{2}}\left[p_{2} \cdot p_{3}+\frac{(y-z) m_{Q}^{2}}{z}\left(1+\frac{4(1-y)}{z}\right)-\frac{1-y}{2 z}\left(s_{1}-5 m_{Q}^{2}\right)\right]+\frac{c_{5}\left(s_{1}, z, y\right)}{s_{3}} \\
& +\frac{c_{6}\left(s_{1}, z, y\right)}{s_{3}^{2}}\left[p_{1} \cdot p_{3}+\frac{z(2-z) / 4-(1-y)(y-z)}{2(y-z / 2)^{2}}\left(s_{1}-m_{Q}^{2}\right)\right]+\frac{d_{1}(\tilde{s}, z)(1-u)\left(\tilde{s}-m_{Q}^{2}\right)}{u t_{1}\left(\tilde{s}-m_{Q}^{2}+t_{1} / z\right)} \\
& +\frac{d_{2}(\tilde{s}, z)(1-u)\left(\tilde{s}-m_{Q}^{2}\right)}{u t_{1}\left[\tilde{s}-m_{Q}^{2}+(2-z) t_{1} / z\right]}+\frac{d_{3}(\tilde{s}, z)(1-u)\left(\tilde{s}-m_{Q}^{2}\right)^{2}}{u t_{1}\left(\tilde{s}-m_{Q}^{2}+t_{1} / z\right)\left[\tilde{s}-m_{Q}^{2}+(2-z) t_{1} / z\right]} \\
& +\frac{d_{4}(\tilde{s}, z)\left(\tilde{s}-m_{Q}^{2}\right)^{2}}{u t_{2}\left[\tilde{s}-m_{Q}^{2}+t_{2} /(1-z)\right]\left[\tilde{s}-m_{Q}^{2}+(2-z) t_{2} /(1-z)\right]}+\frac{d_{5}(\tilde{s}, z)\left(\tilde{s}-m_{Q}^{2}\right)}{u t_{2}\left(\tilde{s}-m_{Q}^{2}+t_{2} /(1-z)\right)}+\frac{h(\tilde{s}, z)\left(\tilde{s}-m_{Q}^{2}\right)^{2}}{t_{2}^{2}}, \\
& +\frac{d_{6}(\tilde{s}, z)\left(\tilde{s}-m_{Q}^{2}\right)}{u t_{2}\left[\tilde{s}-m_{Q}^{2}+(2-z) t_{2} /(1-z)\right]}+\frac{g\left(\tilde{s}-m_{Q}^{2}+t_{2} /(1-z)\right]\left[\tilde{s}-m_{Q}^{2}+(2-z) t_{2} /(1-z)\right]}{u},
\end{aligned}
$$

where $\tilde{s}$ is defined as

$$
\tilde{s}=\left(p_{1}+\tilde{p}\right)^{2}
$$

with

$$
\tilde{p}^{\mu}=p_{2}^{\mu}+p_{3}^{\mu}-\frac{p_{2} \cdot p_{3}}{\left(p_{2}+p_{3}\right) \cdot n} n^{\mu}
$$

Numerically, we have found that the integration of $\left(\mathcal{A}_{\text {real }}-\mathcal{A}_{\mathrm{S}}\right)$ over the phase space is finite in four dimen- sions, which confirms our present choice of the subtraction term $\mathcal{A}_{\mathrm{S}}$. Due to its much simpler structure, following the method of Ref. [28] the phase-space integration over the subtraction term $\mathcal{A}_{\mathrm{S}}$ can be done analytically. The interested reader can turn to Ref. [28] for details.

\section{Renormalization}

The UV divergences in the virtual and real corrections should be canceled through renormalization. The 
counterterm approach is adopted to carry out the renormalization, where the fragmentation function is calculated with the renormalized quark mass $m_{Q}$, the renormalized field $\Psi_{r}$, the renormalized gluon field $A_{r}^{\mu}$, and the renormalized coupling constant $g_{s}$. The renormalized quantities are related to their corresponding bare quantities as

$$
\begin{aligned}
m_{Q}^{0} & =Z_{m} m_{Q}, & \Psi_{0} & =\sqrt{Z_{2}} \Psi_{r}, \\
A_{0}^{\mu} & =\sqrt{Z_{3}} A_{r}^{\mu}, & g_{s}^{0} & =Z_{g} g_{s},
\end{aligned}
$$

where the renormalization constants $Z_{i}=1+\delta Z_{i}$, with $i=m, 2,3, g$, respectively. The quantities $\delta Z_{i}$ are fixed by the renormalized conditions which define a renormalization scheme. The quark field, quark mass, and gluon field are renormalized in the on-mass-shell scheme (OS), whereas the strong coupling constant $g_{s}$ is renormalized in the $\overline{\mathrm{MS}}$ scheme. The expressions for the $\delta Z_{i}$ are

$$
\begin{aligned}
\delta Z_{m}^{\mathrm{OS}}= & -3 C_{F} \frac{\alpha_{s}}{4 \pi}\left[\frac{1}{\epsilon_{\mathrm{UV}}}-\gamma_{E}+\ln \frac{4 \pi \mu_{R}^{2}}{m^{2}}+\frac{4}{3}\right], \\
\delta Z_{2}^{\mathrm{OS}}= & -C_{F} \frac{\alpha_{s}}{4 \pi}\left[\frac{1}{\epsilon_{\mathrm{UV}}}+\frac{2}{\epsilon_{\mathrm{IR}}}-3 \gamma_{E}+3 \ln \frac{4 \pi \mu_{R}^{2}}{m^{2}}+4\right], \\
\delta Z_{3}^{\mathrm{OS}}= & \frac{\alpha_{s}}{4 \pi}\left[\left(\beta_{0}^{\prime}-2 C_{A}\right)\left(\frac{1}{\epsilon_{\mathrm{UV}}}-\frac{1}{\epsilon_{\mathrm{IR}}}\right)\right. \\
& -\frac{4}{3} T_{F}\left(\frac{1}{\epsilon_{\mathrm{UV}}}-\gamma_{E}+\ln \frac{4 \pi \mu_{R}^{2}}{m_{c}^{2}}\right) \\
& \left.-\frac{4}{3} T_{F}\left(\frac{1}{\epsilon_{\mathrm{UV}}}-\gamma_{E}+\ln \frac{4 \pi \mu_{R}^{2}}{m_{b}^{2}}\right)\right], \\
\delta Z_{g}^{\overline{\mathrm{MS}}}= & -\frac{\beta_{0}}{2} \frac{\alpha_{s}}{4 \pi}\left[\frac{1}{\epsilon_{\mathrm{UV}}}-\gamma_{E}+\ln (4 \pi)\right],
\end{aligned}
$$

where $\mu_{R}$ is the renormalization scale, $\beta_{0}=11 C_{A} / 3-$ $4 T_{F} n_{f} / 3$ is the one-loop coefficient of the $\beta$ function in $\mathrm{QCD}$, and $n_{f}$ is the number of active quark flavors. $\beta_{0}^{\prime}=11 C_{A} / 3-4 T_{F} n_{l f} / 3$, and $n_{l f}=3$ is the number of light-quark flavors. For the $S U_{c}(3)$ group, we have $C_{A}=3, C_{F}=4 / 3$, and $T_{F}=1 / 2$.

The operator products in the definition of the fragmentation functions also require renormalization [6,35], whose counterterms in the $\overline{\mathrm{MS}}$ scheme can be written as [24]

$$
\begin{aligned}
D_{Q \rightarrow Q \bar{Q}\left[S_{1}^{[1]}\right]}^{\mathrm{CT}, \text { operator }}(z)= & -\frac{\alpha_{s}}{2 \pi}\left[\frac{1}{\epsilon_{\mathrm{UV}}}-\gamma_{E}+\ln (4 \pi)+\ln \frac{\mu_{R}^{2}}{\mu_{F}^{2}}\right] \\
& \times \int_{z}^{1} \frac{d y}{y} P_{Q Q}(y) D_{Q \rightarrow Q \bar{Q}\left[{ }^{3} S_{1}^{[1]}\right]}^{\mathrm{LO}}(z / y),
\end{aligned}
$$

where $D_{Q \rightarrow Q \bar{Q}\left[^{3} S_{1}^{[1]}\right]}^{\mathrm{LO}}(z)$ denotes the LO fragmentation function in $d$-dimensional space-time.

\section{NUMERICAL RESULTS}

In our numerical calculations the input parameters take the following values:

$m_{c}=1.5 \mathrm{GeV}, \quad m_{b}=4.9 \mathrm{GeV}, \quad m_{Z}=91.1876 \mathrm{GeV}$, $\left|R_{J / \Psi}(0)\right|^{2}=0.810 \mathrm{GeV}^{3}, \quad\left|R_{\Upsilon}(0)\right|^{2}=6.477 \mathrm{GeV}^{3}$,

where the values of $\left|R_{J / \Psi}(0)\right|^{2}$ and $\left|R_{\Upsilon}(0)\right|^{2}$ are taken from the potential-model calculations [36]. For the strong coupling constant, we adopt the two-loop formula,

$\alpha_{s}(\mu)=\frac{4 \pi}{\beta_{0} \ln \left(\mu^{2} / \Lambda_{\mathrm{QCD}}^{2}\right)}\left[1-\frac{\beta_{1} \ln \ln \left(\mu^{2} / \Lambda_{\mathrm{QCD}}^{2}\right)}{\beta_{0}^{2} \ln \left(\mu^{2} / \Lambda_{\mathrm{QCD}}^{2}\right)}\right]$,

where $\beta_{1}=\frac{34}{3} C_{A}^{2}-4 C_{F} T_{F} n_{f}-\frac{20}{3} C_{A} T_{F} n_{f}$ is the two-loop coefficient of the $\beta$ function. According to $\alpha_{s}\left(m_{Z}\right)=$ 0.1185 [37], we obtain $\Lambda_{\mathrm{QCD}}^{n_{f}=5}=0.233$ and $\Lambda_{\mathrm{QCD}}^{n_{f}=4}=$ $0.337 \mathrm{GeV}$. Then we have $\alpha_{s}\left(2 m_{c}\right)=0.259, \alpha_{s}\left(3 m_{c}\right)=$ $0.223, \alpha_{s}\left(2 m_{b}\right)=0.180$, and $\alpha_{s}\left(3 m_{b}\right)=0.164$.

\section{A. The fragmentation functions}

The fragmentation functions for $c \rightarrow J / \Psi$ and $b \rightarrow \Upsilon$ are shown in Figs. 4 and 5, respectively. The initial factorization scale has been set as the minimal invariant mass of the initial $c$ quark or $b$ quark. Two typical values for the renormalization scale $\mu_{R}$ are adopted: one is the threshold energy to create a $c \bar{c}$ or $b \bar{b}$ pair, and the other is the minimal invariant mass of the initial $c$ quark or $b$ quark. Figures 4 and 5 indicate that the renormalization-scale dependence for the LO fragmentation functions is large,

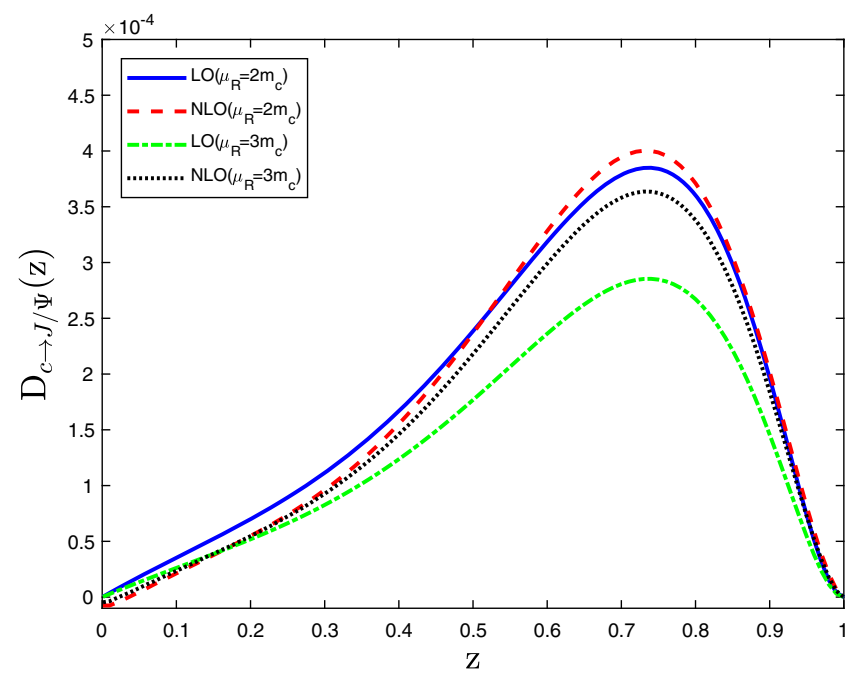

FIG. 4. The fragmentation function $D_{c \rightarrow J / \Psi}\left(z, \mu_{F}, \mu_{R}\right)$ as a function of $z$ at an initial factorization scale $\mu_{F}=3 m_{c}$ up to LO and NLO accuracy, respectively. $\mu_{R}=2 m_{c}$ or $\mu_{R}=3 m_{c}$. 


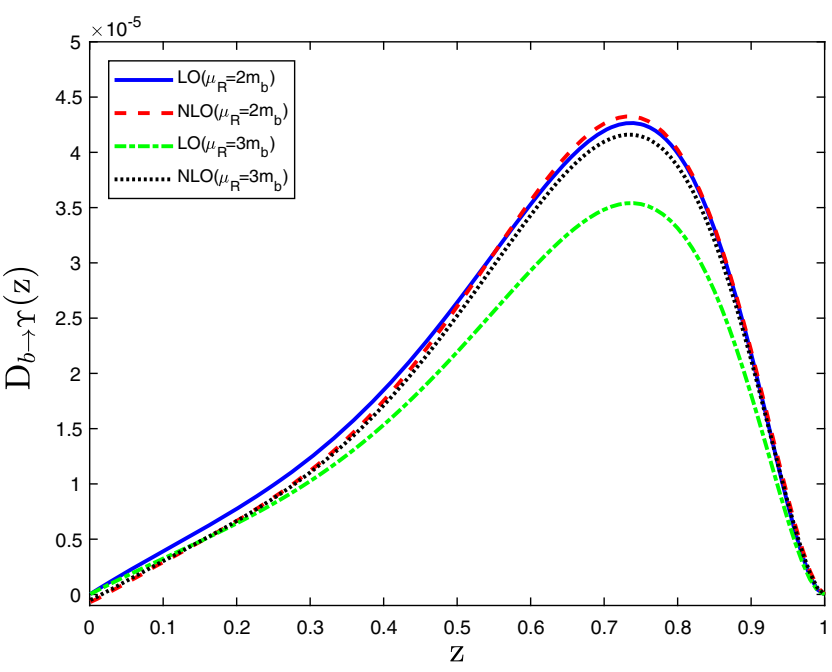

FIG. 5. The fragmentation function $D_{b \rightarrow \Upsilon}\left(z, \mu_{F}, \mu_{R}\right)$ as a function of $z$ at an initial factorization scale $\mu_{F}=3 m_{b}$ up to LO and NLO accuracy. $\mu_{R}=2 m_{b}$ or $\mu_{R}=3 m_{b}$.

and the NLO corrections are very important for reducing the renormalization scale dependence.

For future applications, we use polynomials to fit the NLO fragmentation functions. We write the NLO fragmentation functions in the form

$$
\begin{aligned}
D_{Q \rightarrow H}^{\mathrm{NLO}}\left(z, \mu_{F}\right)= & D_{Q \rightarrow H}^{\mathrm{LO}}(z)\left(1+\frac{\alpha_{s}\left(\mu_{R}\right)}{2 \pi} \beta_{0} \ln \frac{\mu_{R}^{2}}{4 m_{Q}^{2}}\right) \\
& +\frac{\alpha_{s}\left(\mu_{R}\right)}{2 \pi} \ln \frac{\mu_{F}^{2}}{9 m_{Q}^{2}} \int_{z}^{1} \frac{d y}{y} P_{Q Q}(y) D_{Q \rightarrow H}^{\mathrm{LO}}(z / y) \\
& +\frac{\alpha_{s}\left(\mu_{R}\right)^{3}\left|R_{S}(0)\right|^{2}}{m_{Q}^{3}} f(z)
\end{aligned}
$$

where $D_{Q \rightarrow H}^{\mathrm{LO}}(z)$ is the $\mathrm{LO}$ fragmentation function given in Eq. (22). For $c \rightarrow J / \Psi$, we have

$$
\begin{aligned}
f(z)= & -9.01726 z^{10}+18.22777 z^{9}+16.11858 z^{8} \\
& -82.54936 z^{7}+106.57565 z^{6}-72.30107 z^{5} \\
& +28.85798 z^{4}-6.70607 z^{3}+0.84950 z^{2} \\
& -0.05376 z-0.00205 .
\end{aligned}
$$

For $b \rightarrow \Upsilon$, we have

$$
\begin{aligned}
f(z)= & -14.00334 z^{10}+46.94869 z^{9}-55.23509 z^{8} \\
& +16.69070 z^{7}+22.09895 z^{6}-26.85003 z^{5} \\
& +13.41858 z^{4}-3.50293 z^{3}+0.46758 z^{2} \\
& -0.03099 z-0.00226 .
\end{aligned}
$$

Using the fragmentation function, we can obtain two useful quantities, i.e., the fragmentation probability $(P)$ and the averaged energy fraction $(\langle z\rangle)$, which are defined as
TABLE I. The fragmentation probability and the average value of the energy fraction for $c \rightarrow J / \Psi . \mu_{F}=3 m_{c}$.

\begin{tabular}{lcccc}
\hline \hline & $P^{\mathrm{LO}} \times 10^{4}$ & $P^{\mathrm{NLO}} \times 10^{4}$ & $\langle z\rangle^{\mathrm{LO}}$ & $\langle z\rangle^{\mathrm{NLO}}$ \\
\hline$\mu_{R}=2 m_{c}$ & 1.88 & 1.86 & 0.62 & 0.63 \\
$\mu_{R}=3 m_{c}$ & 1.40 & 1.72 & 0.62 & 0.63 \\
\hline \hline
\end{tabular}

TABLE II. The fragmentation probability and the averaged energy fraction for $b \rightarrow \Upsilon$. $\mu_{F}=3 m_{b}$.

\begin{tabular}{ccccc}
\hline \hline & $P^{\mathrm{LO}} \times 10^{5}$ & $P^{\mathrm{NLO}} \times 10^{5}$ & $\langle z\rangle^{\mathrm{LO}}$ & $\langle z\rangle^{\mathrm{NLO}}$ \\
\hline$\mu_{R}=2 m_{b}$ & 2.09 & 2.05 & 0.62 & 0.63 \\
$\mu_{R}=3 m_{b}$ & 1.73 & 1.99 & 0.62 & 0.63 \\
\hline \hline & $P_{Q \rightarrow H}=\int_{0}^{1} D_{Q \rightarrow H}\left(z, \mu_{F}\right) d z$, \\
& \\
$\langle z\rangle=\frac{\int_{0}^{1} z D_{Q \rightarrow H}\left(z, \mu_{F}\right) d z}{\int_{0}^{1} D_{Q \rightarrow H}\left(z, \mu_{F}\right) d z}$.
\end{tabular}

The fragmentation probabilities and the averaged energy fractions for $c \rightarrow J / \Psi$ and $b \rightarrow \Upsilon$ are shown in Tables I and II, respectively.

The fragmentation functions shown in Figs. 4 and 5 are for $\mu_{F}=3 m_{Q}$. The fragmentation functions at any other factorization scales can be obtained through the DGLAP equation. To apply the NLO fragmentation functions to the production of heavy quarkonia at a $Z$ factory, we present the fragmentation functions for $\mu_{F}=m_{Z}$ in Figs. 6 and 7, which are obtained by using the fragmentation functions at the initial value $\mu_{F}=3 m_{Q}$. For definiteness, we set the renormalization scale $\mu_{R}=2 m_{Q}$. In doing the numerical calculation, the DGLAP equation is solved through the Mellin transformation [38,39], and the NLO expression

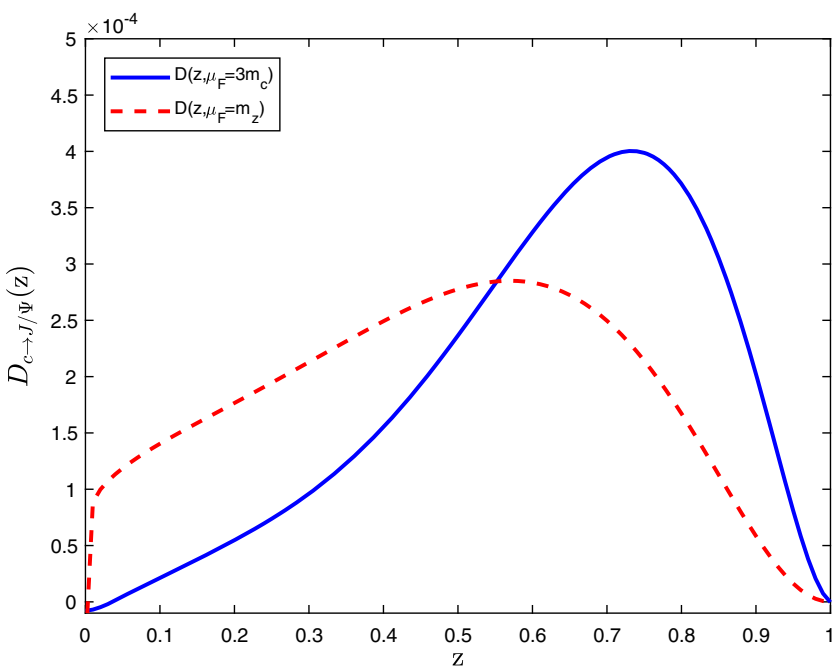

FIG. 6. The NLO fragmentation function $D_{c \rightarrow J / \Psi}$ as a function of $z$ for $\mu_{F}=3 m_{c}$ and $\mu_{F}=m_{Z}$, respectively. $\mu_{R}=2 m_{c}$. 


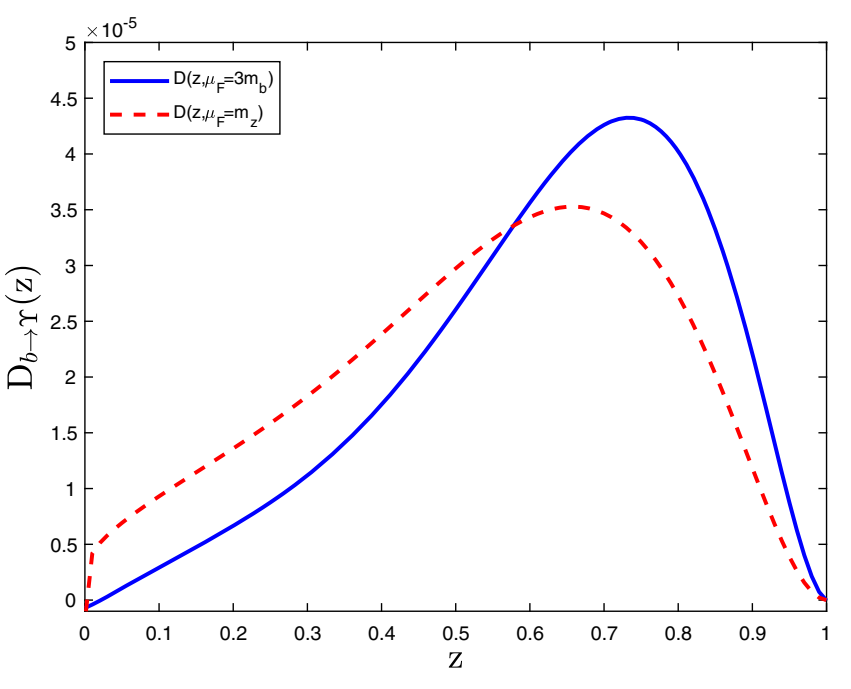

FIG. 7. The NLO fragmentation function $D_{b \rightarrow \Upsilon}$ as a function of $z$ for $\mu_{F}=3 m_{b}$ and $\mu_{F}=m_{Z}$, respectively. $\mu_{R}=2 m_{b}$.

for $P_{Q Q}$ is used as the evolution kernel. Figures 6 and 7 show that the peaks of the fragmentation functions become lower and shift to smaller $z$ values for a larger factorization scale, leading to a smaller value for the averaged energy fraction $\langle z\rangle$. For example, we obtain $\left.\langle z\rangle^{\mathrm{NLO}+\mathrm{NLL}}\right|_{\mu_{F}=m_{Z}}=$ 0.47 for $D_{c \rightarrow J / \Psi}$ and $\left.\langle z\rangle^{\mathrm{NLO}+\mathrm{NLL}}\right|_{\mu_{F}=m_{Z}}=0.54$ for $D_{b \rightarrow r}$ (where NLL stands for next-to-leading logarithm).

\section{B. $J / \Psi$ and $\Upsilon$ production at a $Z$ factory}

As an application, we apply the NLO fragmentation functions of $c \rightarrow J / \Psi$ and $b \rightarrow \Upsilon$ to the production of $J / \Psi$ and $Y$ at a super $Z$ factory.

A Chinese group has raised the proposal of constructing a high-luminosity $e^{+} e^{-}$collider in China-the so-called super $Z$ factory [40] — which is similar to the GigaZ program suggested by the Internal Linear Collider Collaboration $[41,42]$ but with an even higher luminosity. As for this purposed super $Z$ factory, an $e^{+} e^{-}$collider would run at energies around the mass of the $Z^{0}$-boson resonance and with a high luminosity up to $\mathcal{L}=10^{34}$ $10^{36} \mathrm{~cm}^{-2} \mathrm{~s}^{-1}$. Due to the $Z^{0}$-boson resonance effect, large numbers of $J / \Psi$ and $\Upsilon$ events can be produced, thus providing a good platform for studying the properties of $J / \Psi$ and $\Upsilon$.

In this case, the factorization formula for the production of $J / \Psi$ or $\Upsilon$ can be written as

$$
\begin{aligned}
\frac{d \sigma_{e^{+} e^{-} \rightarrow H+X}}{d z}= & \sum_{i} \int_{z}^{1} \frac{d y}{y} \frac{d \hat{\sigma}_{e^{+} e^{-} \rightarrow i+X}\left(y, \mu_{F}\right)}{d y} . \\
& D_{i \rightarrow H}\left(z / y, \mu_{F}\right),
\end{aligned}
$$

where the energy fraction $z$ is defined as $z \equiv 2 p \cdot k / k^{2}, p$ is the momentum of the produced quarkonium, and $k$ is the sum of the momenta of the initial electron and positron.
Up to the NLO level, the parton $i$ may be a heavy quark or a heavy antiquark. For quarkonium production, the fragmentation function $D_{\bar{Q} \rightarrow H}$ is the same as $D_{Q \rightarrow H}$.

Due to the fact that the coefficient functions $d \hat{\sigma} / d y$ are independent of the species of the produced hadron, they can be extracted by applying the $\mathrm{pQCD}$ factorization formula to the production of an on-shell heavy quark $(Q)$ or heavy antiquark $(\bar{Q})[43,44]$. The expression for the coefficient function $d \hat{\sigma} / d y$ in the $\overline{\mathrm{MS}}$ scheme up to the NLO level has been given in Refs. [45,46], e.g.,

$$
\begin{aligned}
& \frac{d \hat{\sigma}_{e^{+} e^{-} \rightarrow Q+X}^{\mathrm{LO}}}{d y}\left(y, \mu_{F}\right) \\
& =\sigma_{e^{+} e^{-} \rightarrow Q \bar{Q}}^{\mathrm{LO}}\left[\delta(1-y)+\frac{\alpha_{s}\left(\mu_{R}\right)}{2 \pi}\left(P_{Q Q}(y) \ln \frac{s}{\mu_{F}^{2}}+C(y)\right)\right],
\end{aligned}
$$

where $\sigma_{e^{+} e^{-} \rightarrow Q \bar{Q}}^{\mathrm{LO}}$ is the $\mathrm{LO}$ cross section for $Q \bar{Q}$ production. And in the massless limit $m_{Q} \rightarrow 0,{ }^{1}$ we have

$$
\begin{aligned}
\sigma_{e^{+} e^{-} \rightarrow Q \bar{Q}}^{\mathrm{LO}}= & \frac{4 \pi N_{c} \alpha^{2}}{3 s}\left[e_{e}^{2} e_{Q}^{2}+2 e_{e} v_{e} e_{Q} v_{Q} \rho_{1}(s)\right. \\
& \left.+\left(v_{e}^{2}+a_{e}^{2}\right)\left(v_{Q}^{2}+a_{Q}^{2}\right) \rho_{2}(s)\right],
\end{aligned}
$$

where $e_{f}$ is the electric charge of the fermion $f$,

$$
\begin{gathered}
v_{f}=\left(T_{3 f}-2 e_{f} \sin ^{2} \theta_{w}\right) /\left(2 \sin \theta_{w} \cos \theta_{w}\right), \\
a_{f}=T_{3 f} /\left(2 \sin \theta_{w} \cos \theta_{w}\right)
\end{gathered}
$$

are the vector and axial-vector couplings of the fermion $f$ to the $Z$ boson, $T_{3 f}$ is the third component of the weak isospin of the fermion $f, \theta_{w}$ is the weak mixing angle, and the propagator functions are

$$
\begin{aligned}
& \rho_{1}(s)=\frac{s\left(s-m_{Z}^{2}\right)}{\left(s-m_{Z}^{2}\right)^{2}+m_{Z}^{2} \Gamma_{Z}^{2}}, \\
& \rho_{2}(s)=\frac{s^{2}}{\left(s-m_{Z}^{2}\right)^{2}+m_{Z}^{2} \Gamma_{Z}^{2}} .
\end{aligned}
$$

The function $C(y)$ in the massless limit $m_{Q} \rightarrow 0$ takes the form

$$
\begin{aligned}
C(y)= & C_{F}\left\{\left(\frac{2 \pi^{2}}{3}-\frac{9}{2}\right) \delta(1-y)-\frac{3}{2}\left(\frac{1}{1-y}\right)_{+}\right. \\
& +2\left(\frac{\ln (1-y)}{1-y}\right)_{+}-(1+y)[2 \ln y+\ln (1-y)] \\
& \left.+4 \frac{\ln y}{1-y}+\frac{5}{2}-\frac{3 y}{2}\right\} .
\end{aligned}
$$

\footnotetext{
${ }^{1}$ Because the coefficient function $d \hat{\sigma} / d y$ is infrared safe, we can take the limit $m_{Q} \rightarrow 0$ to do our calculation, which will introduce a small error of $\mathcal{O}\left(m_{Q}^{2} / s\right)$.
} 
For comparison, we adopt three strategies to calculate the differential cross sections $d \sigma / d z$ using the fragmentation approach. We denote them as "Frag, LO", "Frag, NLO", and "Frag, NLO + NLL", respectively. For the case of "Frag, LO," the differential cross sections are calculated as

$$
\begin{aligned}
\frac{d \sigma_{e^{+} e^{-} \rightarrow H+X}^{\mathrm{LO}}}{d z}= & 2 \int_{z}^{1} \frac{d y}{y} \frac{d \hat{\sigma}_{e^{+} e^{-} \rightarrow Q+\bar{Q}}^{\mathrm{LO}}}{d y}\left(y, \mu_{F}\right) \\
& \cdot D_{Q \rightarrow H}^{\mathrm{LO}}\left(z / y, \mu_{F}\right) \\
= & 2 \sigma_{e^{+} e^{-} \rightarrow Q+\bar{Q}}^{\mathrm{LO}} \cdot D_{Q \rightarrow H}^{\mathrm{LO}}\left(z, \mu_{F}\right),
\end{aligned}
$$

where the factor of 2 comes from the contribution of the $\bar{Q}$ fragmentation which is the same as that of $Q$ fragmentation. $D_{Q \rightarrow H}^{\mathrm{LO}}(z)$ denotes the $\mathrm{LO}$ fragmentation function given in Eq. (22). In the second equation, we have used the fact that $d \hat{\sigma}_{e^{+} e^{-} \rightarrow Q+\bar{Q}}^{\mathrm{LO}} / d y=\sigma_{e^{+} e^{-} \rightarrow Q+\bar{Q}}^{\mathrm{LO}} \delta(1-y)$. For the case of "Frag, NLO", the differential cross sections are calculated as

$$
\begin{aligned}
\frac{d \sigma_{e^{+} e^{-} \rightarrow H+X}^{\mathrm{NLO}}}{d z}= & 2 \int_{z}^{1} \frac{d y}{y} \frac{d \hat{\sigma}_{e^{+} e^{-} \rightarrow Q+X}^{\mathrm{NLO}}}{d y}\left(y, \mu_{F}\right) \\
& \times D_{Q \rightarrow H}^{\mathrm{NLO}}\left(z / y, \mu_{F}\right) .
\end{aligned}
$$

In the above calculation, the factorization and renormalization scales are set as $\mu_{F}=3 m_{Q}$ and $\mu_{R}=2 m_{Q}$. For the case of "Frag, NLO + NLL", the differential cross sections are calculated as

$$
\begin{aligned}
\frac{d \sigma_{e^{+} e^{-} \rightarrow H+X}^{\mathrm{NLO}+\mathrm{NLL}}}{d z}= & 2 \int_{z}^{1} \frac{d y}{y} \frac{d \hat{\sigma}_{e^{+} e^{-} \rightarrow Q+X}^{\mathrm{NLO}}}{d y}\left(y, \mu_{F}\right) \\
& \times D_{Q \rightarrow H}\left(z / y, \mu_{F}\right),
\end{aligned}
$$

where the factorization scale and renormalization scale are set as $\mu_{F}=\mu_{R}=m_{Z}$, the fragmentation functions

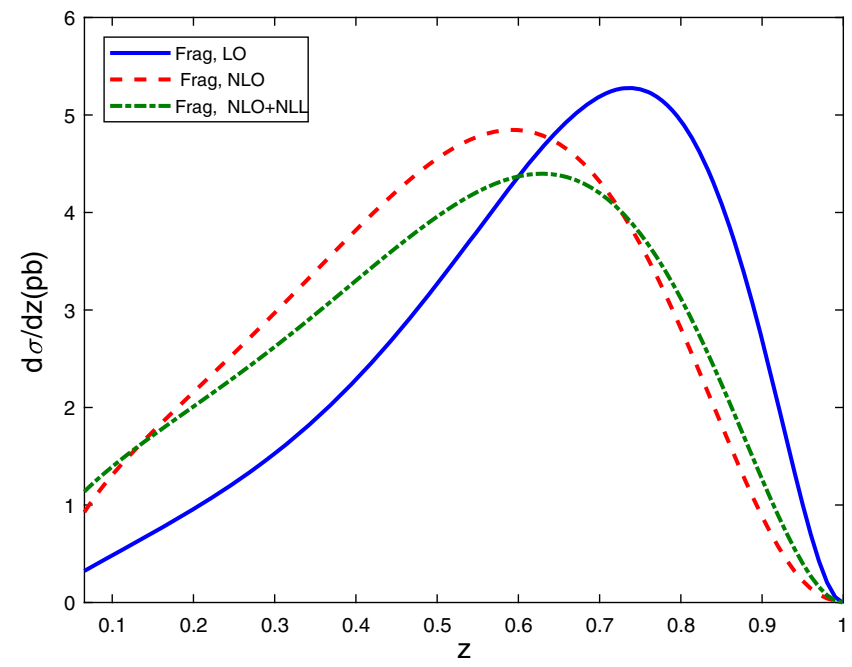

FIG. 8. The differential cross section $d \sigma / d z$ of $e^{+} e^{-} \rightarrow J / \Psi+$ $X$ at the $Z$ pole using the fragmentation approach.

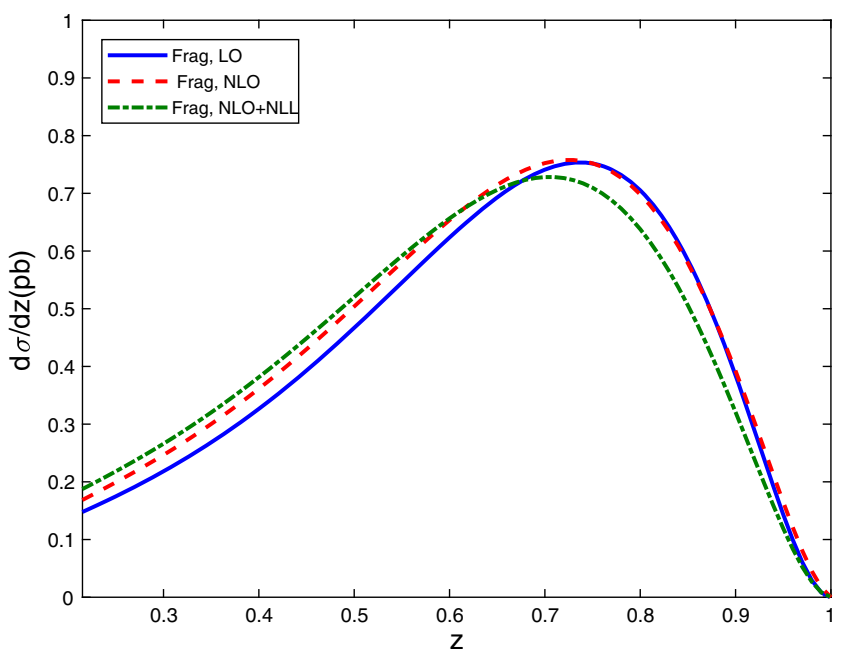

FIG. 9. The differential cross section $d \sigma / d z$ of $e^{+} e^{-} \rightarrow \Upsilon+X$ at the $Z$ pole using the fragmentation approach.

$D_{Q \rightarrow H}\left(z, \mu_{F}=m_{Z}\right)$ are obtained by solving the DGLAP evolution equation [i.e., Eq. (4)], and the initial fragmentation functions $D_{Q \rightarrow H}^{\mathrm{NLO}}\left(z, \mu_{F}=3 m_{Q}\right)$ with $\mu_{R}=2 m_{Q}$ are used as the boundary condition. In this way, the large log terms such as $\ln \left(m_{Q}^{2} / m_{Z}^{2}\right)$ are resummed up to NLL accuracy.

We present the differential cross sections $d \sigma / d z$ for the production of $J / \Psi$ and $\Upsilon$ via the fragmentation approach in Figs. 8 and 9. In drawing the figures, we have omitted the $\gamma-\gamma$ and $\gamma-Z$ contributions, which are quite small compared with the dominant $Z-Z$ contribution around the $Z$ pole. Figures 8 and 9 show how the NLO terms and the leading and next-to-leading logarithms affect the predictions. For $J / \Psi$ production the NLO contribution is significant, and after including the NLO terms the distribution becomes softer and the value of $z$ corresponding to the peak value of the distribution becomes smaller. For $\Upsilon$ production the NLO contribution is relatively small compared with the $J / \Psi$ case.

Integrating the differential cross sections $d \sigma / d z$ over $z$, we can obtain the total cross sections for $J / \Psi$ and $\Upsilon$ production at the $Z$ factory, which can be simplified as

$$
\sigma(H)=2 P_{Q \rightarrow H} \int_{0}^{1} \frac{d \hat{\sigma}_{e^{+} e^{-} \rightarrow Q+X}}{d y}\left(y, \mu_{F}\right)
$$

where $P_{Q \rightarrow H}$ is the fragmentation probability for $Q$ into the quarkonium $H$. The results are presented in Table III, which

TABLE III. Total cross sections (in pb) of the production channels $e^{+} e^{-} \rightarrow J / \Psi+X$ and $e^{+} e^{-} \rightarrow \Upsilon+X$ at the $Z$ pole.

\begin{tabular}{lccc}
\hline \hline & Frag, LO & Frag, NLO & Frag, NLO + NLL \\
\hline$J / \Psi$ & 2.58 & 2.77 & 2.65 \\
$\Upsilon$ & 0.368 & 0.382 & 0.377 \\
\hline \hline
\end{tabular}




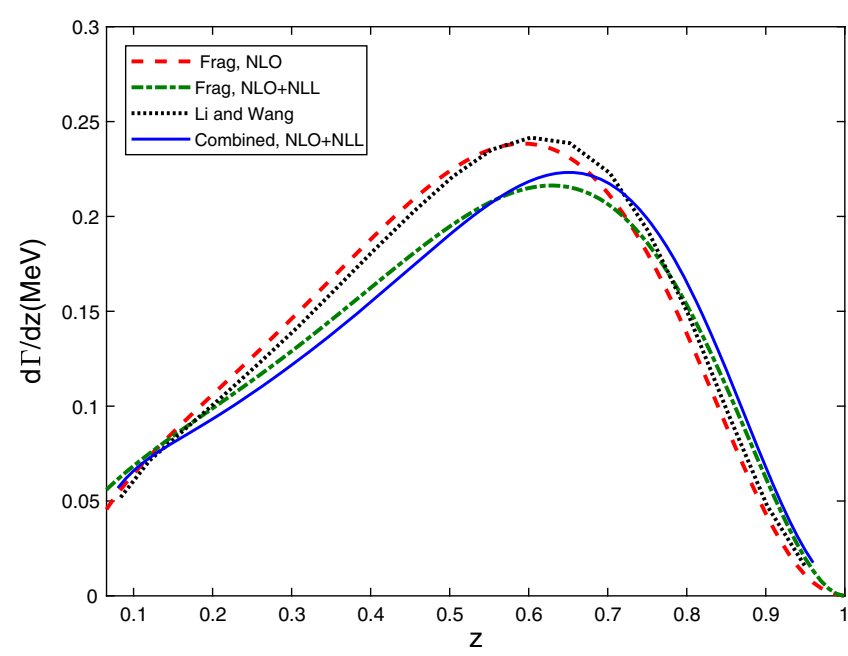

FIG. 10. The differential width $d \Gamma / d z$ of $Z \rightarrow J / \Psi+X$.

shows that the NLO corrections enhance the total cross section by $\sim 7 \%$ for $J / \Psi$ and $\sim 4 \%$ for $\Upsilon$.

\section{A comparison of $J / \Psi$ and $\Upsilon$ production via the $Z$ decays up to the NLO level}

As a final remark, we compare the NLO results under the fragmentation function approach and the complete pQCD approach. The complete NLO pQCD calculations of $Z \rightarrow J / \Psi+X$ and $Z \rightarrow \Upsilon+X$ have been done in Ref. [5]. We present the differential decay widths $d \Gamma / d z$ for $Z \rightarrow J / \Psi+X$ and $Z \rightarrow \Upsilon+X$ in Figs. 10 and 11, in which all of the input parameters are taken from Ref. [5] and the results denoted by "Li and Wang" are results under the complete NLO calculation. The results denoted by "Combined, NLO + NLL" are a combination of the results from the complete pQCD approach (taken from Ref. [5]) and the results from the fragmentation

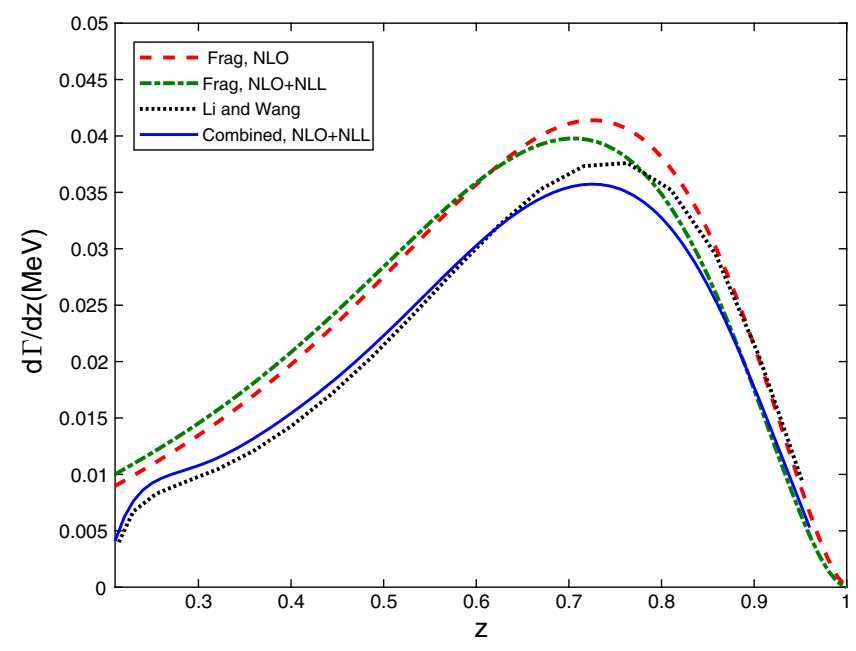

FIG. 11. The differential width $d \Gamma / d z$ of $Z \rightarrow \Upsilon+X$. approach, i.e., $d \Gamma /\left.d z\right|_{\text {Combined,NLO+NLL }}=d \Gamma /\left.d z\right|_{\text {Li and Wang }}+$ $\left(d \Gamma /\left.d z\right|_{\text {Frag,NLO+NLL }}-d \Gamma /\left.d z\right|_{\text {Frag,NLO }}\right)$. For the case of $J / \Psi$ production at the $Z$ factory, Fig. 10 shows that the fragmentation contributions dominate the decay width, since the "Frag, NLO" shape is very close to the complete NLO one. Moreover, the total decay widths are $\Gamma(Z \rightarrow J / \Psi+$ $X)\left.\right|_{\text {Frag, NLO }}=136 \mathrm{keV},\left.\quad \Gamma(Z \rightarrow J / \Psi+X)\right|_{\text {Frag,NLO+NLL }}=$ $130 \mathrm{keV},\left.\quad \Gamma(Z \rightarrow J / \Psi+X)\right|_{\mathrm{Li} \text { and } \mathrm{Wang}}=136 \mathrm{keV}, \quad$ and $\left.\Gamma(Z \rightarrow J / \Psi+X)\right|_{\text {Combined,NLO+NLL }}=130 \mathrm{keV}$. Figure 11 shows that the "Frag, NLO" result is larger than the result from the complete NLO calculation in the small- $z$ region, and thus the fragmentation approximation for $\Upsilon$ production is not as good as in the $J / \Psi$ case. This is because the $b$-quark mass is larger than the $c$-quark mass, and the power correction in $m_{b}^{2} / s$ for the case of $\Upsilon$ is larger than for the case of $J / \Psi$. However, the combined result counts both the large power correction and the large logarithms, which gives a good prediction. Moreover, the total decay widths are $\left.\Gamma(Z \rightarrow \Upsilon+X)\right|_{\text {Frag, NLO }}=20.9 \mathrm{keV}, \quad \Gamma(Z \rightarrow J / \Upsilon+$ $X)\left.\right|_{\text {Frag, NLO+NLL }}=20.6 \mathrm{keV},\left.\Gamma(Z \rightarrow J / \Upsilon+X)\right|_{\text {Li and Wang }}=$ $17.38 \mathrm{keV}, \quad$ and $\left.\quad \Gamma(Z \rightarrow J / \Upsilon+X)\right|_{\text {Combined,NLO+NLL }}=$ $17.08 \mathrm{keV}$.

\section{SUMMARY}

In the present paper, we have calculated the fragmentation function for a heavy quark into heavy quarkonium, e.g., $c \rightarrow J / \Psi$ or $b \rightarrow \Upsilon$, up to the NLO level. Our present results are complementary to previous works on the fragmentation function of a gluon into heavy quarkonia, which is pQCD calculable due to the fact that the gluon should be hard enough to form a heavy quarkantiquark pair.

Our results show that the NLO correction is important for suppressing the renormalization scale uncertainty and to achieve a reliable fragmentation prediction. Our calculations are based on the gauge-invariant definition of the fragmentation function suggested by Collins and Soper. To avoid large logarithms appearing in the perturbative series of the fragmentation function, we first derived the fragmentation function at an initial (reasonable) factorization scale $\mu_{F}=3 m_{Q}$, and then ran to any factorization scale with the help of the DGLAP evolution equation. This treatment, in effect, resums the large logarithms and provides a reliable prediction. Thus, for the cases when the fragmentation dominates the quarkonium productions or decays, our present calculated fragmentation functions will be of great help for a more precise pQCD prediction. As an application, we have applied the obtained fragmentation functions to the production of $J / \Psi$ and $\Upsilon$ at a super $Z$ factory. The shape of the $J / \Psi$ distribution changes significantly by introducing the NLO corrections, and the total cross section increases by $\sim 7 \%$. The shape of the $\Upsilon$ distribution changes slightly by introducing the NLO corrections, and the total cross section increases only $\sim 4 \%$. 


\section{ACKNOWLEDGMENTS}

This work was supported in part by the Natural Science Foundation of China under Grants No. 11625520, No. 11847222, No. 11847301, No. 11675239, and No. 11535002, and by the Fundamental Research Funds for the Central Universities under Grant No. 2019CDJDWL0005.

[1] G. T. Bodwin, E. Braaten, and G. P. Lepage, Rigorous QCD analysis of inclusive annihilation and production of heavy quarkonium, Phys. Rev. D 51, 1125 (1995); Erratum, Phys. Rev. D 55, 5853(E) (1997).

[2] N. Brambilla et al., Heavy quarkonium: Progress, puzzles, and opportunities, Eur. Phys. J. C 71, 1534 (2011) and references therein.

[3] N. Brambilla et al., Heavy Quarkonium Physics, CERN2005-005 20 June 2005, arXiv:hep-ph/0412158.

[4] G. Altarelli and G. Parisi, Asymptotic freedom in parton language, Nucl. Phys. B126, 298 (1977); Yu. L. Dokshitzer, Calculation of the structure functions for deep inelastic scattering and $e^{+} e^{-}$annihilation by perturbation theory in quantum chromodynamics, Sov. Phys. JETP 46, 641 (1977); L. N. Lipatov, The parton model and perturbation theory, Yad. Fiz. 20, 181 (1974); V. Gribov and L. Lipatov, $e^{+} e^{-}$pair annihilation and deep inelastic e $\mathrm{p}$ scattering in perturbation theory, Sov. J. Nucl. Phys. 15, 675 (1972).

[5] R. Li and J.-X. Wang, Next-to-leading-order QCD correction to inclusive $J / \Psi(\Upsilon)$ production in $Z^{0}$ decay, Phys. Rev. D 82, 054006 (2010).

[6] J. C. Collins and D. E. Soper, Parton distribution and decay functions, Nucl. Phys. B194, 445 (1982).

[7] G. C. Nayak, J.-W. Qiu, and G. Sterman, Fragmentation, nonrelativistic and NNLO factorization analysis in heavy quarkonium production, Phys. Rev. D 72, 114012 (2005).

[8] Z.-B. Kang, J.-W. Qiu, and G. Sterman, Factorization and quarkonium production, Nucl. Phys. B, Proc. Suppl. 214, 39 (2011).

[9] Z.-B. Kang, J.-W. Qiu, and G. Sterman, Heavy Quarkonium Production and Polarization, Phys. Rev. Lett. 108, 102002 (2012).

[10] S. Fleming, A. K. Leibovich, T. Mehen, and I. Z. Rothstein, The systematics of quarkonium production at the LHC and double parton fragmentation, Phys. Rev. D 86, 094012 (2012).

[11] S. Fleming, A. K. Leibovich, T. Mehen, and I. Z. Rothstein, Anomalous dimensions of the double parton fragmentation functions, Phys. Rev. D 87, 074022 (2013).

[12] S. Mandelstam, Proc. R. Soc. 233, 248 (1955).

[13] C.-H. Chang and Y.-Q. Chen, The Production of $B_{c}$ or $\bar{B}_{c}$ associated with two heavy quark jets in $Z^{0}$ boson decay, Phys. Rev. D 46, 3845 (1992); Errtum, Phys. Rev. D 50, 6013(E) (1994).

[14] E. Braaten, K. Cheung, and T. C. Yuan, QCD fragmentation functions for $B_{c}$ or $B_{c}^{*}$ production, Phys. Rev. D 48, R5049 (1993).

[15] Y.L. Dokshitzer, Calculation of the structure functions for deep inelastic scattering and $e^{+} e^{-}$annihilation by perturbation theory in quantum chromodynamics, Zh. Eksp. Teor. Fiz. 73, 1216 (1977); Sov. Phys. JETP 46, 641 (1977).

[16] V. N. Gribov and L. N. Lipatov, Deep inelastic ep scattering in perturbation theory, Yad. Fiz. 15, 781 (1972); Sov. J. Nucl. Phys. 15, 438 (1972).

[17] G. Altarelli and G. Parisi, Asymptotic freedom in parton language, Nucl. Phys. B126, 298 (1977).

[18] G. Curci, W. Furmanski, and R. Petronzio, Evolution of parton densities beyond leading order: The nonsinglet case, Nucl. Phys. B175, 27 (1980).

[19] W. Furmanski and R. Petronzio, Singlet parton densities beyond leading order, Phys. Lett. 97B, 437 (1980).

[20] E. G. Floratos, D. A. Ross, and C. T. Sachrajda, Higher order effects in asymptotically free gauge theories. 2. Flavor singlet Wilson operators and coefficient functions, Nucl. Phys. B152, 493 (1979); A. Gonzalez-Arroyo and C. Lopez, Second order contributions to the structure functions in deep inelastic scattering. 3. The singlet case, Nucl. Phys. B166, 429 (1980); E. G. Floratos, C. Kounnas, and R. Lacaze, Higher order QCD effects in inclusive annihilation and deep inelastic scattering, Nucl. Phys. B192, 417 (1981).

[21] E. Braaten, K. Cheung, and T.C. Yuan, $Z^{0}$ decay into charmonium via charm quark fragmentation, Phys. Rev. D 48, 4230 (1993).

[22] J.-P. Ma, Calculating fragmentation functions from definitions, Phys. Lett. B 332, 398 (1994).

[23] R. Sepahvand and S. Dadfar, NLO corrections to c- and b-quark fragmentation into $J / \Psi$ and $\Upsilon$, Phys. Rev. D 95, 034012 (2017).

[24] P. Artoisenet and E. Braaten, Gluon fragmentation into quarkonium at next-to-leading order, J. High Energy Phys. 04 (2015) 121.

[25] P. Artoisenet and E. Braaten, Gluon fragmentation into quarkonium at next-to-leading order using FKS subtraction, J. High Energy Phys. 01 (2019) 227.

[26] F. Feng and Y. Jia, Next-to-leading-order QCD corrections to gluon fragmentation into ${ }^{1} S_{0}^{(1,8)}$ quarkonia, arXiv: 1810.04138.

[27] P. Zhang, C.-Y. Wang, X. Liu, Y.-Q. Ma, C. Meng, and K.-T. Chao, Semi-analytical calculation of gluon fragmentation into ${ }^{1} S_{0}^{1,8]}$ quarkonia at next-to-leading order, J. High Energy Phys. 04 (2019) 116.

[28] X.-C. Zheng, C.-H. Chang, T.-F. Feng, and X.-G. Wu, The QCD NLO fragmentation functions from $c$ or $\bar{b}$ quark to $B_{c}$ or $B_{c}^{*}$ meson and their application, arXiv: 1901.03477 . 
[29] R. Mertig, M. Bohm, and A. Denner, Feyn CalcComputer-algebraic calculation of Feynman amplitudes, Comput. Phys. Commun. 64, 345 (1991).

[30] V. Shtabovenko, R. Mertig, and F. Orellana, New developments in FeynCalc 9.0, Comput. Phys. Commun. 207, 432 (2016).

[31] F. Feng, \$Apart: A generalized mathematica apart function, Comput. Phys. Commun. 183, 2158 (2012).

[32] A. V. Smirnov, Algorithm FIRE-Feynman Integral REduction, J. High Energy Phys. 10 (2008) 107.

[33] T. Hahn and M. Perez-Victoria, Automatized one loop calculations in four-dimensions and D-dimensions, Comput. Phys. Commun. 118, 153 (1999).

[34] M. Beneke and V. A. Smirnov, Asymptotic expansion of Feynman integrals near threshold, Nucl. Phys. B522, 321 (1998).

[35] A. H. Mueller, Cut vertices and their renormalization: A generalization of the Wilson expansion, Phys. Rev. D 18, 3705 (1978).

[36] E. J. Eichten and C. Quigg, Mesons with beauty and charm: Spectroscopy, Phys. Rev. D 49, 5845 (1994); and references therein.

[37] C. Patrignani et al. (Particle Data Group), Review of Particle Physics, Chin. Phys. C 40, 100001 (2016).
[38] G. Curci, W. Furmanski, and R. Petronzio, Evolution of parton densities beyond leading order: The nonsinglet case, Nucl. Phys. B175, 27 (1980).

[39] B. Mele and P. Nason, The fragmentation function for heavy quarks in QCD, Nucl. Phys. B361, 626 (1991).

[40] J. P. Ma and Z. X. Zhang (The Super Z-Factory Group), Preface, Sci. Chin. Phys. Mech. Astron. 53, 1947 (2010).

[41] G. Aarons et al. (ILC Collaboration), International Linear Collider Reference Design Report 2 Physics at the ILC (2007).

[42] J. Erler S. Heinemeyerb, W. Hollikc, G. Weigleind, and P. M. Zerwasb, Physics impact of GigaZ, Phys. Lett. B 486, 125 (2000).

[43] J. C. Collins and G. Sterman, Soft partons in QCD, Nucl. Phys. B185, 172 (1981).

[44] J. C. Collins, D. E. Soper, and G. Sterman, Factorization of hard process in QCD, Adv. Ser. Direct High Energy Phys. 5, 1 (1989).

[45] R. Baier and K. Fey, Finite corrections to quark fragmentation functions in perturbative QCD, Z. Phys. C 2, 339 (1979).

[46] G. Altarelli, R. K. Ellis, G. Martinelli, and S. Y. Pi, Processes involving fragmentation functions beyond the leading order in QCD, Nucl. Phys. B160, 301 (1979). 